Kazazian and Freedman (J. Biol. Chem., 243, 6446; 1968) have made these observations the basis of a pretty demonstration of the current picture of protein synthesis, and also of a projected method for obtaining messenger RNA for a single species of haemoglobin chain, $\alpha$ or $\beta$. The critical facts are that the rabbit $\alpha$-chain has three isoleucine residues, two of them (numbers 10 and 17) near the $\mathrm{N}$-terminus (from which protein synthesis proceeds), whereas the $\beta$-chain has only one, at position 112, only twenty-four residues from the C-terminus. What one then expects to happen is that $\alpha$-chain synthesis, in the presence of the analogue, will be substantially blocked at the first isoleucine, so that only small polysomes, bearing short polypeptide chain, will result. Synthesis of the $\beta$-chain, on the other hand, will cease only when the product is already 111 residues long. At this point, because the chains cannot be completed or released, ribosomes will pile up on the messenger, like railway wagons in a siding, and large polysomes will be anticipated. Expectations are in fact borne out, the polysome distribution showing on the one hand an increase in species with two and three ribosomes, and on the other the appearance of polysomes of ten to fourteen ribosomes. By labelling with ${ }^{14} \mathrm{C}$, and hydrolysis with trypsin, followed by peptide mapping, the peptides originating from the $\alpha$ and $\beta$-chains could be identified and counted. The specific activity of $\alpha$ and $\beta$-peptides in a control showed that the number of $\alpha$ and $\beta$-chains usually initiated was nearly enough equal throughout the polysome distribution. Sampling the heavy and light polysomes in the analogue-treated system, it was found instead that the heavy fraction contained 86 per cent of $\beta$-chain material, whereas the lightest fraction had 68 per cent of $\alpha$-chains. Further, the total number of ribosomes attached to the $\alpha$ and $\beta$-chain messengers can be determined from the ratio of $\mathrm{N}$-terminal $\alpha$ to $\beta$-peptides (as each participating ribosome will bear one peptide chain). As expected, there is a large excess of $\beta$-chain ribosomes. Thus all predictions based on the present view of the synthesis mechanism are fulfilled. Moreover, this approach clearly indicates a strategy for the preparation of gene-specific messengers in isolation.

Temmerman and Leblen (Biochim. Biophys. Acta, 174, 544; 1969) have found that when rabbit reticulocyte ribosomes are dissociated by treatment with EDTA, the rapidly labelled fraction of the RNA sediments relatively rapidly and appears to be associated with ribosomal protein. The manner of this association is undetermined.

Another difficulty in isolating messenger is the likelihood of degradation by endogenous nuclease. Farkas and Marks (J. Biol. Chem., 243, 6464; 1968) have now partially purified and characterized this nuclease from rabbit reticulocytes and it appears to be specific for pyrimidines. A particularly striking observation is the action of haemin as a specific inhibitor of this enzyme, and not of pancreatic or $T 1$ ribonucleases. This immediately provokes a line of reasoning whereby the sequestration of haemin by newly synthesized globin liberates the nuclease activity; the nuclease then goes to work on the surrounding RNA, and globin synthesis is interrupted. The low haemin concentration causes haemin biosynthesis to be renewed, the nuclease is consequently inhibited once more, and so on. The mature red cell makes no protein and contains no ribosomes, these having presumably been annihilated by the nuclease, which will be active when all the haem is in the form of haemoglobin. It is conceivable that this mechanism may underlie the oscillations in the rate of protein synthesis in reticulocytes, just reported by Tepper et al. (FEBS Lotters, 2, 217; 1969)

\section{CELL BIOLOGY \\ A Chromosome Attachment Site}

\section{from our Cell Biology Correspondent}

A COMPLEx of bacterial cell membrane, bacterial chromosome and nascent RNA which may well prove to contain the hitherto elusive bacterial chromosome attachment site, has been isolated from Bacillus megatherium by Tremblay, Daniels and Schaecter at Tufts University, Boston. In bacteria, the chromosome is believed to be attached to a specific binding site on the cell membrane rather than lying free in the cytoplasm. The idea is that this attachment site provides a structural basis for chromosome segregation, analogous in function to the mitotic spindle, and possibly for DNA replication as well. When the attachment site and the chromosome have divided, segregation is believed to take place as the cell wall elongates and the attachment sites move apart, each dragging a chromosome with it.

In the past, several groups have attempted to isolate this hypothetical DNA-membrane complex as a discrete subcellular fraction, but all these studies have suffered from the same drawback. Cell membrane and DNA were sedimented together so that it has always been impossible to rule out adventitious non-specific association between DNA and membrane material. Tremblay, Daniels and Schaecter, however, now report (J. Mol. Biol., 40,65; 1969) an ingenious method for fractionating fragments of bacterial membrane which appears to yield the chromosome still attached to the specific attachment site.

They have exploited the ability of portions of the cell membrane, but not the chromosomes, to adhere to crystals of a detergent Sarkosyl (sodium lauroyl sarcosinate) in the presence of $\mathrm{Mg}^{++}$ions. Essentially their method consists of lysing Bacillus megatherium with sarkosyl and banding the lysate in a sucrose gradient containing $\mathrm{Mg}^{++}$ions, which induces the crystallization of the sarkosyl in situ. Ten to thirty per cent of the cell membrane, more than 90 per cent of the DNA and about 75 per cent of the nascent RNA in the cells occur associated with the crystals. Because nucleic acids along have no affinity for sarkosyl, the simplest inference is that the DNA and RNA in the complex is there because it is still attached to fragments of the membrane containing the chromosome attach. ment site. The RNA, in turn, is presumably attached to the DNA from which it was being transcribed.

Electron micrographs of the complex reveal membrane from which emerge long DNA molecules. It is unlikely that these complexes are adventitious, especially because, when the same technique is used to fractionate $E$. coli infected with $\mathrm{T}_{4}$ phage, the $E$. coli DNA occurs in the complexes whereas the phage DNA does not. It seems therefore that the material necessary for a detailed investigation of some of the obscurer processes of bacterial chromosome replication and segregation is now available. 